\title{
Comment on: "The consequences of gynaecological cancer in patients and their partners from the sexual and psychological perspective"
}

\author{
Salvatore Giovanni Vitale ${ }^{1}$, Valentina Lucia La Rosa ${ }^{2}$, Agnese Maria Chiara Rapisarda ${ }^{3}$, \\ Antonio Simone Laganà ${ }^{1}$
}

${ }^{1}$ Unit of Gynaecology and Obstetrics, Department of Human Pathology in Adulthood and Childhood "G. Barresi”, University of Messina, Messina, Italy

${ }^{2}$ Unit of Psychodiagnostics and Clinical Psychology, University of Catania, Catania, Italy

${ }^{3}$ Department of General Surgery and Medical Surgical Specialties, University of Catania, Catania, Italy

\section{Dear Editor,}

We read with great interest the review by lżycki et al. [1] about the sexual, psychological, and social consequences of gynaecological cancer in patients and their partners.

We agree that the impact of gynaecological cancer on mental health, sexual functioning, and emotional wellbeing of affected women and their partners is considerable, and it is important to investigate this topic in order to ensure that gynaecological cancer patients receive optimal care [2].

Although we appreciated the reported methodology, we would like to take this opportunity to point out several elements that would let us further realise the results of this study.

In our opinion, it would have been more appropriate to further differentiate sexual and psychological consequences according to the type of gynaecological cancer and the type of treatment. Indeed, the literature about this topic underlined that the type of cancer (endometrial, cervical, ovarian, or vulvar cancer) and the type of treatment (such as simple or radical hysterectomy, radiotherapy, and/or chemotherapy) may have different effects on the sexual health and quality of life of affected women [2, 3]. For example, ovarian cancer is responsible for more deaths than any other cancer of the female reproductive system, and the majority of patients present with advanced-stage disease [3-5]; therefore, it is associated with more serious consequences from a psychological point of view. Moreover, it has been demonstrated that radical surgery and radiation therapy may affect sexual functioning more negatively than less radical surgery [2, 6-8].

For these reasons, we believe that it would be appropriate to take into account several factors, such as type and stage of cancer and treatment modality, in or- der to better understand the impact of these variables on the sexual and psychological well-being of patients.

Nonetheless, it is important to underline that most of the studies done on this topic used very heterogeneous samples for age, disease stage, and treatment, so it is necessary to conduct further studies with more homogeneous samples in order to obtain more reliable and reproducible data.

We appreciate the section of the review devoted to the consequences of gynaecological cancer for the partners of the affected women. Indeed, the impact of cancer on the partner's sexual function, partner's relationship satisfaction, and general well-being between the couple are less investigated in the literature about this topic, so further studies about this topic are needed.

In conclusion, this review confirms that gynaecological cancer patients are at risk of developing sexual and psychological problems, so it is essential to provide adequate counselling and support for these women and their partners.

Specifically, we believe that it is important to identify patients who are particularly at risk of developing sexual and psychological problems. It would be appropriate to make an assessment including the sexual function before cancer, current sexual activity, and how cancer has influenced sexual health and relationship with the partner. For this purpose, the Brief Index of Sexual Functioning for Women [9] or the Female Sexual Function Index (FSFI) [10] could be used. The assessment should include also the presence of psychiatric comorbidities and psychological diseases, such as anxiety and depression, through the use of validate psychodiagnostic tests.

Another important point to be highlighted is the need for adequate training of healthcare professionals in order to better provide information and support to 
patients during the communication of diagnosis and throughout the therapeutic process.

The aim is to reduce as much as possible the impact of cancer on the sexual and psychological functioning of patients and their partners.

\section{References}

1. Iżycki D, Woźniak K, lżycka N. Consequences of gynecological cancer in patients and their partners from the sexual and psychological perspective. Menopause Rev 2016; 15: 112-116.

2. Huffman LB, Hartenbach EM, Carter J, et al. Maintaining sexual health throughout gynecologic cancer survivorship: A comprehensive review and clinical guide. Gynecol Oncol 2016; 140: 359-368.

3. Domenici L, Palaia I, Giorgini M, et al. Sexual Health and Quality of Life Assessment among Ovarian Cancer Patients during Chemotherapy. Oncology 2016; 91: 205-210.

4. Bellia A, Vitale SG, Laganà AS, et al. Feasibility and surgical outcomes of conventional and robot-assisted laparoscopy for early-stage ovarian cancer: a retrospective, multicenter analysis. Arch Gynecol Obstet 2016; 294: 615-622.

5. Vitale SG, Marilli I, Lodato $M$, et al. The role of cytoreductive surgery in advanced-stage ovarian cancer: a systematic review. Updates Surg 2013; 65: 265-270.

6. Greimel ER, Winter R, Kapp KS, et al. Quality of life and sexual functioning after cervical cancer treatment: a long-term follow-up study. Psychooncology 2009; 18: 476-482.

7. Vitale SG, Valenti G, Gulino FA, et al. Surgical treatment of high stage endometrial cancer: current perspectives. Updates Surg 2016; 68: 149154.

8. Rossetti D, Vitale SG, Gulino FA, et al. Concomitant chemoradiation treatment in selected stage I endometrioid endometrial cancers. Eur J Gynaecol Oncol 2016; 37: 657-661.

9. Rosen R, Brown C, Heiman J, et al. The Female Sexual Function Index (FSFI): a multidimensional self-report instrument for the assessment of female sexual function. J Sex Marital Ther 2000; 26: 191-208.

10. Taylor JF, Rosen RC, Leiblum SR. Self-report assessment of female sexual function: psychometric evaluation of the Brief Index of Sexual Functioning for Women. Arch Sex Behav 1994; 23: 627-643. 